\title{
A New Approach for Creating Polymer Hydrogels with Regions of Distinct Chemical, Mechanical, and Optical Properties
}

\author{
Stephen J. Banik, Neville J. Fernandes, Peter C. Thomas, and Srinivasa R. Raghavan* \\ Department of Chemical \& Biomolecular Engineering, University of Maryland, College Park, Maryland 20742-2111, United States
}

ABSTRACT: We describe a new approach to creating hybrid polymer hydrogels that comprise two different gel types (gel 1 and gel 2) juxtaposed in predetermined zones or patterns and with the unique properties of each gel being retained. The key to our approach is to ensure that the viscosities of the pregel mixtures are high when they are brought into contact and subsequently polymerized; this limits the diffusion at gel/gel interfaces. The final gel appears as a single, homogeneous, transparent material with smooth, robust interfaces between the dissimilar zones. However, its hybrid nature is revealed by specific

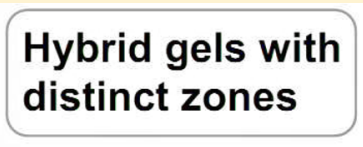
. In one example, we use the same monomer, $N, N$ dimethylacrylamide, for gels 1 and 2, but gel 1 is cross-linked by a chemical cross-linker while gel 2 is cross-linked by laponite nanoparticles. In this case, when the hybrid gel is immersed in a mixed solution of cationic and anionic dyes, gel 2 selectively absorbs the cationic dye due to the strong affinity of the nanoparticles in it for cationic species. Additionally, a mechanical test on the above hybrid shows that the gel 2 region is stronger and much more extensible than the gel 1 region. We also make use of the fact that the gel 2 (high-laponite) regions are optically birefringent relative to gel 1 (low or no laponite). This allows us to embed a pattern ("message") of gel 2 within gel 1 . While the hybrid gel appears featureless under white light, the hidden "message" becomes visible when the gel is viewed under crossed polarizers. The overall approach described here can be extended in myriad ways for the generation of gels possessing new and unique properties.

\section{INTRODUCTION}

Polymer hydrogels are three-dimensional networks of polymer chains cross-linked by chemical or physical bonds and swollen in water. ${ }^{1,2}$ Their wide-ranging and tunable properties have propelled them to the forefront of many current technological applications. Typically, these gels are formed by free-radical polymerization of a monomer such as $\mathrm{N}$-isopropylacrylamide (NIPA) in water using a chemical cross-linker such as $N, N^{\prime}$ methylenebis(acrylamide) (BIS). ${ }^{3,4}$ NIPA gels are known for their temperature-dependent response: they shrink when heated above $\sim 32{ }^{\circ} \mathrm{C} .{ }^{3}$ Other monomers or monomer combinations have been used to create a variety of stimuliresponsive gels including those that shrink upon cooling, ${ }^{1,2}$ those that shrink in certain aqueous solvent mixtures, ${ }^{1}$ or in response to $\mathrm{pH}^{5}$ or ionic strength. ${ }^{6}$ Many potential applications have arisen for stimuli-responsive hydrogels including in drug delivery, ${ }^{7,8}$ tissue engineering, ${ }^{9,10}$ and as biomaterials. ${ }^{8}$ As research on hydrogels has progressed, scientists have been looking to engineer gels with a combination of properties (e.g., gels that are responsive to more than one stimulus, etc.) This challenge has been largely approached from the standpoint of polymer chemistry, i.e., in suitably engineering the molecular structure of the monomer and/or the cross-linker.

An alternative way to engineer new gels is to physically combine multiple gel components into a single material, while still retaining the unique features of each component. This cannot be achieved by simply mixing two different monomer solutions before polymerization-in that case, the monomers would be copolymerized into a single network that would not retain the properties of each individual gel. Instead, the approach that is usually adopted to combine different gels into the same material is the one pioneered by $\mathrm{Hu}$ et al. in the late 1990 s. $^{11,12}$ In this approach, the first monomer is allowed to completely polymerize, and then it is soaked in a pregel solution of the second monomer. Thereby, the two monomers do not mix and the final gel retains the character of each component. This approach has been used to create patterns of one gel in another ${ }^{12}$ and also to impart shape memory effects to the combined material. ${ }^{11}$ However, the method does have its limitations in that it is best suited to patterning in two dimensions and also that the components do not remain fully separated (the first gel is still mixed with the second monomer and thereby partially interwoven with the second gel).

In this paper, we present a new approach for combining dissimilar gels into one whole material while fully preserving the unique character of each individual gel. The key to our approach is that we bring dissimilar pregel mixtures into contact when their viscosities are sufficiently high and thereafter polymerize the hybrid. The high viscosities eliminate convective mixing and slow down diffusive mixing at gel/gel interfaces. Our approach allows the two individual gel components to be spatially juxtaposed in a desired fashion in the hybrid gel. The

Received: May 1, 2012

Revised: June 20, 2012

Published: July 10, 2012 
final gel visually appears as a single, homogeneous, transparent material. Interfaces between the component gels in the hybrid are smooth, i.e., not apparent on visual inspection; moreover, the interfaces are highly robust and mechanical failure does not occur at these locations.

The utility of our approach is demonstrated in this paper by three distinct examples where we leverage the chemical, mechanical, and optical heterogeneity of our hybrid gels. The hybrid gels described here comprise two different gel types (gel 1 and gel 2) that share the same monomer but use different cross-linkers. While gel 1 is cross-linked by a chemical crosslinker such as BIS, gel 2 is cross-linked by nanoparticles of a synthetic clay from the laponite family. ${ }^{13}$ The use of laponite nanoparticles as substitutes for conventional chemical crosslinkers was first demonstrated by Haraguchi et al. about a decade ago. ${ }^{14,15}$ The resulting gels have mostly physical rather than covalent cross-links, ${ }^{16,17}$ but compared to conventional gels, they have much higher mechanical strength and extensibility. ${ }^{17,18}$ Recently, we showed that laponite-crosslinked gels can separate cationic dyes from solution due to the strong chemical affinity of laponite particles for cationic species. ${ }^{19}$ This result is extended to our hybrid gels. We demonstrate that the laponite-cross-linked (gel 2) regions of our hybrids exhibit a strong affinity for cationic dyes, which is not present in gel 1 regions. In addition, gel 2 regions are shown to have distinct mechanical properties as well as optical (birefringence) properties. An application of the latter is shown where gel 2 (high-laponite) regions are embedded as a hidden pattern or "message" within a matrix of gel 1 (low-laponite). The "message" is revealed either when the hybrid gel is viewed under crossed polarizers or when heated. In closing, we note that our overall approach is simple and versatile; it can be easily extended in myriad ways to create new gels with unusual or unique properties.

\section{EXPERIMENTAL SECTION}

Materials. The monomers $N, N$-dimethylacrylamide (DMAA), $N$ isopropylacrylamide (NIPA), and $N, N^{\prime}$-methylenebis(acrylamide) (BIS), the initiator potassium persulfate (KPS), and the accelerator $N, N, N^{\prime}, N^{\prime}$-tetramethylethylenediamine (TEMED) were all purchased from Sigma-Aldrich. The inorganic clay (a synthetic hectorite) Laponite XLG was obtained from Southern Clay Products. The cationic dye methylene blue (MB) was purchased from Sigma-Aldrich while the anionic dye 5(6)-carboxyfluorescein (CF) was purchased from ACROS. All materials were used as received.

Basic Gel Preparation. Two types of cross-linkers were used to produce the polymer networks in the hybrid gels: the tetrafunctional acrylamide derivative BIS for chemical cross-links and laponite clay particles for physical cross-links. Gels were prepared using a $15 \mathrm{~g}$ water basis. First, nitrogen gas was bubbled through the deionized water (18 $\mathrm{M} \Omega$, Millipore-spec) for $3 \mathrm{~h}$ prior to use to remove any dissolved oxygen. Monomer was then dissolved in the water at a $1 \mathrm{M}$ concentration, following which cross-linker was added. In the case of BIS, the concentration used was $2.2 \mathrm{~mol} \%$ (with respect to the monomer). In the case of laponite, the content ranged between 3.3 and $6.4 \mathrm{wt} \%$ (with respect to total solution weight), and the particles had to be added slowly to avoid clumping. The solution was stirred for $\sim 10$ min on a magnetic stirrer plate, and its final $\mathrm{pH}$ was around 9.5. The accelerator (TEMED) and initiator (KPS) were then added at concentrations each of $0.015 \mathrm{~g} / \mathrm{mL}$. At this point polymerization began, and it was allowed to proceed at room temperature in a nitrogen-only environment for $20 \mathrm{~h}$.

DMAA/LAP-DMAA/BIS Hybrid Gel. The hybrid gel for dye separation and mechanical tests was a $5 \mathrm{~mm}$ diameter rod, $\sim 7 \mathrm{~cm}$ long. One half of the rod was BIS-cross-linked and the other half was laponite-cross-linked-both portions using DMAA as the monomer.
After preparing the pregel solutions as described above, the DMAA/ LAP mixture was pipetted into a $5 \mathrm{~mm}$ diameter glass vial. Because the viscosity of this mixture was quite high, the DMAA/BIS pregel solution could be immediately pipetted on top of the DMAA/LAP mixture without the two pregels mixing-a single, easy step for combining the two gels. After $20 \mathrm{~h}$ of polymerization, the vial was broken and the gel hybrid was removed and rinsed.

NIPA/LAP(6.4)-NIPA/LAP(3.3) Hybrid Gel. The hybrid gels in which a pattern is embedded (hidden) were made with NIPA as the monomer and laponite of different concentrations as the cross-linker. A high-laponite pregel was created with 6.4 wt \% laponite, and a lowlaponite pregel was made with $3.3 \mathrm{wt} \%$ laponite. In the case of the high-laponite pregel, the particles had to be added to the monomer solution very slowly along with vigorous mixing by magnetic stirring as well as using a vortex mixer. The resulting viscous mixture was then centrifuged to remove trapped bubbles. The mixture was then transferred to a cake pipetting bag in order to hand-write the pattern/message. After the message was written, the $3.3 \mathrm{wt} \%$ pregel was poured around until the message was just covered. The hybrid was then placed in an oxygen-free environment and polymerized for $20 \mathrm{~h}$.

NIPA/LAP(4.9)-NIPA/LAP(3.3) Hybrid Gel. Another NIPAbased hybrid gel with a hidden pattern was made using laponite of moderate concentration (4.9 wt \%) to write the pattern/message. The corresponding pregel mixture was not as viscous as the $6.4 \mathrm{wt} \%$ and did not require a cake pipetting bag. Instead, a simple disposable pipet was used to write the message. Once again, after the message was written, the low-laponite $(3.3 \mathrm{wt} \%)$ pregel was immediately poured around it, and the hybrid was polymerized as above.

\section{RESULTS AND DISCUSSION}

Figure 1 shows a hybrid gel created as a rod of diameter $5 \mathrm{~mm}$ and length $7 \mathrm{~cm}$. The DMAA monomer was used for both of the components of the hybrid. The top half of the hybrid, termed DMAA/LAP, was cross-linked using laponite nanoparticles, as shown schematically in the upper portion of Figure

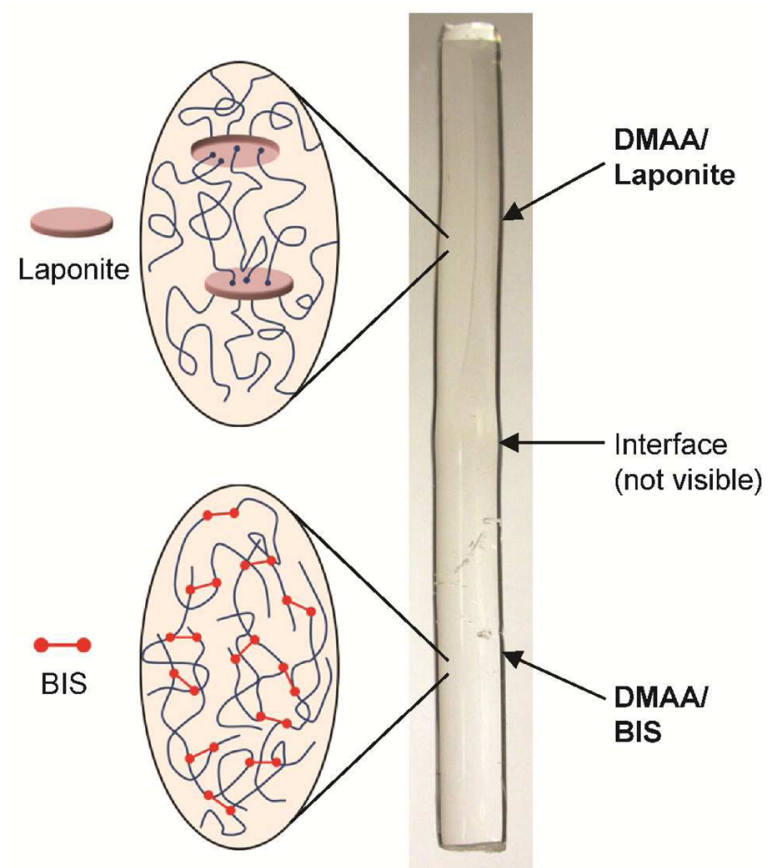

Figure 1. A hybrid gel rod ( $5 \mathrm{~mm}$ diameter, $7 \mathrm{~cm}$ long) made from DMAA monomer with a laponite-cross-linked portion (top half) and a BIS-cross-linked portion (bottom half). Schematics of the two regions of the hybrid are shown on the left. Note that the interface between the regions is smooth and hence not visible. 

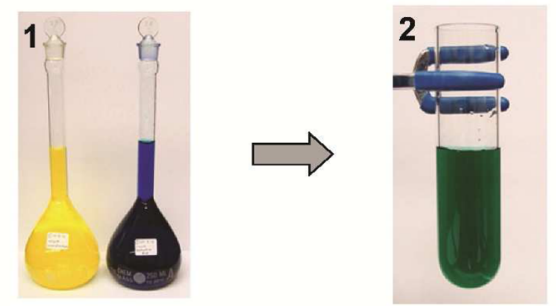

50/50 mixture of CF (yellow) and MB (blue) dyes
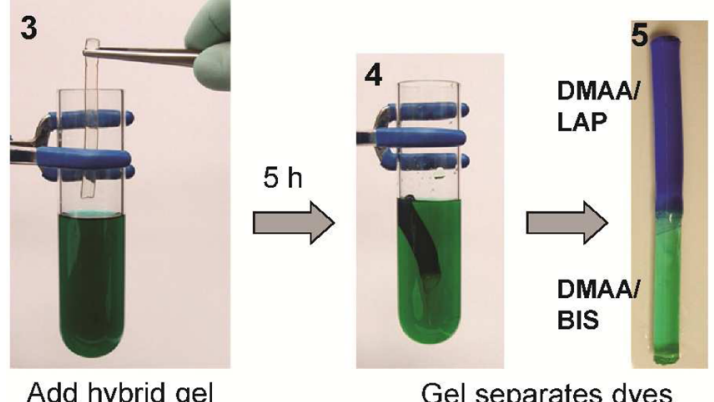

Gel separates dyes

Figure 2. Chemical differences between zones of the hybrid gel. A mixture of the cationic dye $\mathrm{MB}$ and the anionic dye (CF) is made (panels 1,2), and the gel is immersed in it (panel 3). Because of the affinity of laponite particles for cationic solutes, the DMAA/LAP portion of the gel (top half) selectively absorbs the MB and separates it from solution (panels 4,5 ). Some of the CF is absorbed by the DMAA/BIS portion (bottom half) of the gel.

1. The laponite particles are discs of diameter $\sim 25 \mathrm{~nm}$ and thickness $\sim 1 \mathrm{~nm} .{ }^{13}$ The polymer chains are expected to extend from one face of such a disk and terminate at another, as indicated in the schematic. ${ }^{16,17}$ The bottom half of the hybrid, termed DMAA/BIS, was cross-linked using the conventional BIS cross-linker, shown as red dumbbells in the lower schematic. The polymer chains are covalently cross-linked by BIS molecules within this region. Note that the interface between the two regions of the hybrid gel is not discernible in the photograph. Thus, the overall gel looks to be a single, homogeneous mass.

It is worth reiterating the key aspects of our procedure for creating the above hybrid. The central idea arose out of our observation that adding a few wt $\%$ of laponite particles to a monomer solution (pregel) makes the mixture quite viscous. This is not surprising because laponite is well-known for its ability to thicken and gel water by physical interactions of the particles. ${ }^{13}$ The viscous nature implies that when two laponitecontaining pregels are brought into contact, they will not mix at their interface. In fact, as long as at least one of the pregel solutions contains laponite and is therefore viscous, the viscosity of the other pregel need not be high when the two pregels are brought into contact. This concept can be extended to pregels not containing laponite by noting that cross-linking of a pregel (e.g., DMAA/BIS) in the presence of KPS initiator and TEMED accelerator at room temperature causes the viscosity of the pregel to slowly, but substantially, increase at the initial stages of the polymerization. We then simply have to wait a short amount of time (minutes) such that at least one of the pregels becomes noticeably viscous prior to contact with the other.

For the hybrid gel in Figure 1, we first added the DMAA/ LAP pregel into a $5 \mathrm{~mm}$ vial and then the DMAA/BIS pregel

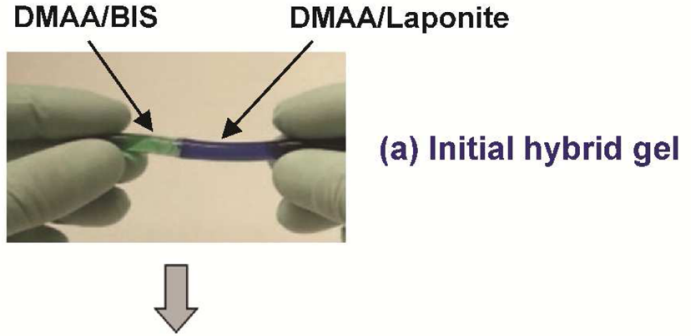

(b) Gel being stretched

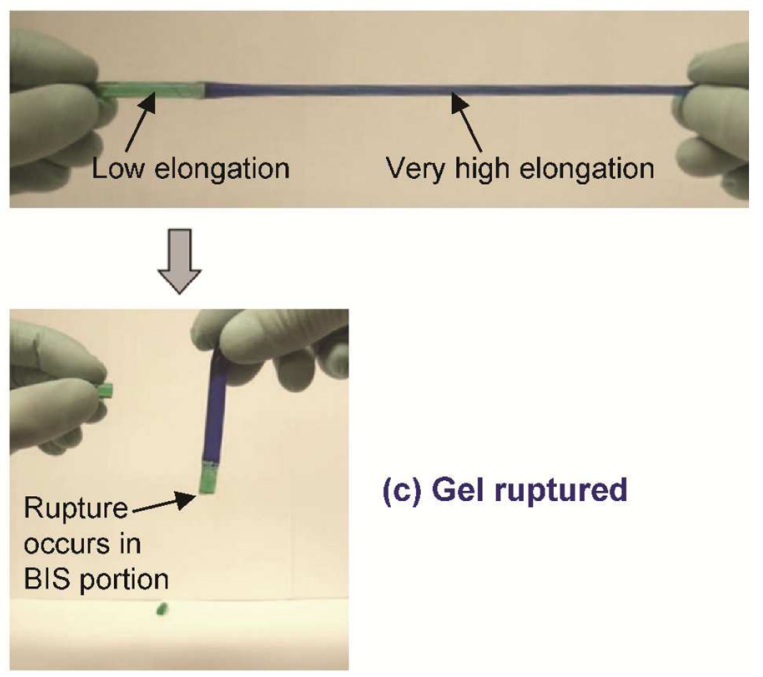

Figure 3. Mechanical differences between zones of the hybrid gel. (a) A hybrid gel with DMAA/BIS and DMAA/LAP regions is shown in its initial, unstretched state. The absorbed dyes from Figure 2 help to distinguish the two regions. (b) When stretched, the BIS region elongates slightly whereas the LAP region gets highly elongated. (c) With continued stretching, the gel eventually ruptures. The break point always occurs in the DMAA/BIS portion, showing that the gel strength is much higher for DMAA/LAP than for the DMAA/BIS. Also, the rupture does not occur at the interface between the LAP and BIS regions, which indicates that the gel/gel interface is highly robust.

on top. Both mixtures were sufficiently viscous (consistency of weak pastes) at the time of contact. This ensured that the two zones of the hybrid did not undergo convective mixing and moreover, even diffusive mixing was quite weak during the remainder of the polymerization. Note that if instead the two pregels were largely polymerized (i.e., converted to gels) at the time of contact, there would be no diffusive mixing at all at the interface, in which case the interface would be very weak. In the current case, there is still some weak diffusive mixing, which ensures that the zones of the hybrid are seamlessly melded and with their interface practically indiscernible.

We now contrast the properties of the two zones of the hybrid gel. First, we focus on the differences in ionic dye absorption from solution. For this, the cationic dye $\mathrm{MB}$ and the anionic dye $\mathrm{CF}$ were combined (Figure 2, panel 1), producing a dark blue/green solution (panel 2), with the final concentration of each dye in this solution being $50 \mu \mathrm{M}$. The above hybrid gel ( $\sim 2 \mathrm{~g}$ in mass) was placed in this solution (panel 3). After $\sim 5 \mathrm{~h}$ with no stirring, we note that the cationic $\mathrm{MB}$ dye is preferentially absorbed by the DMAA/LAP portion of the hybrid, due to which that portion assumes a blue color (panel 5). In contrast, the DMAA/BIS portion is seen to have absorbed some of both dyes and consequently has a light green color (panel 5). These differences are due to the anionic faces 
(a) Message written with NIPA/LAP (6.4\%) pre-gel

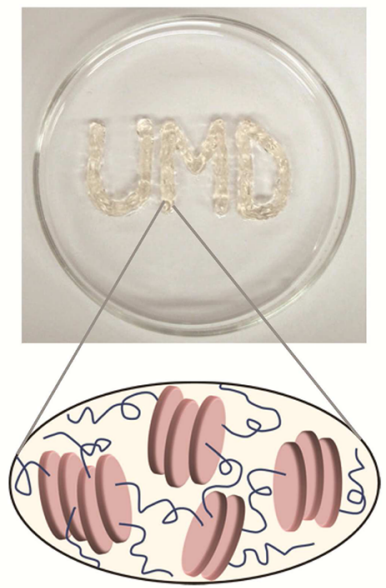

$\mathrm{C}_{\text {Lap }}>\mathrm{C}_{\text {crit }}$ in message $\Rightarrow$ particles stack and align, leading to birefringence (b) NIPA/LAP $(3.3 \%)$ pre-gel added around, polymerized: message hidden in hybrid gel
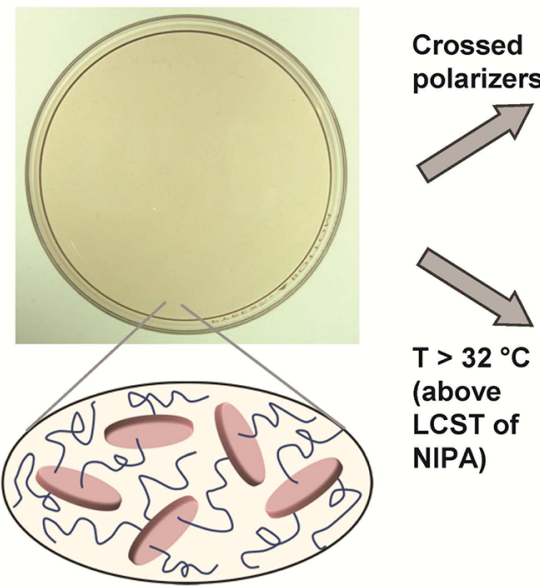

(above

LCST of NIPA) (c) Message revealed due to its birefringence
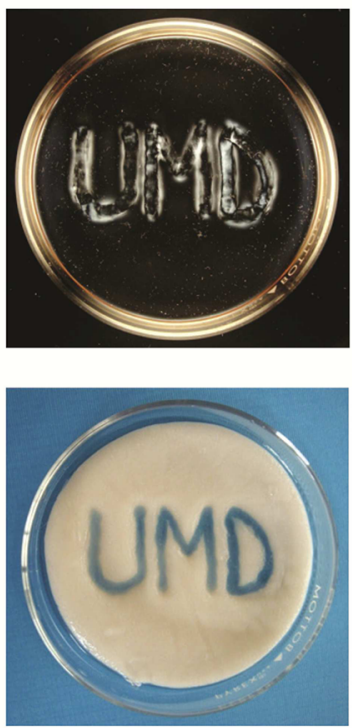

particles remain unaligned, no birefringence (d) Message revealed due to its lower opacity

Figure 4. Embedding a hidden pattern or "message" in a hybrid gel. (a) The message "UMD" is written with a pregel of NIPA/LAP (6.4 wt \%). At this higher concentration, the laponite particles stack and align, leading to birefringence. The message is surrounded by a pregel of NIPA/LAP (3.3 wt \%). At this lower concentration of laponite, the particles are unaligned and there is no birefringence. (b) After the entire system is polymerized, the gel appears homogeneous in the Petri dish and the message cannot be seen under normal light. (c) When the gel is viewed under crossed polarizers, the message region lights up due to its birefringence. (d) The message can also be revealed under normal light if the gel is heated above the LCST of NIPA. In this case, the message region is relatively transparent while the surrounding gel is opaque (the underlying blue background beneath the Petri dish can be seen through the message).

of laponite disks having a strong affinity for cationic solutes. ${ }^{13,19}$ As a result, laponite-bearing gels can effectively separate a cationic dye from a mixture of cationic and anionic dyes. ${ }^{19,20}$ Indeed, the DMAA/LAP zone of the hybrid gel in the present case is able to extract much of the MB from the solution-thus, the final solution has a much lower MB concentration (and a greener color, panel 4) compared to the original solution. Overall, Figure 2 shows that the zones of the hybrid have different chemical properties due to the cross-linkers chosen for the zones. Similarly, distinct chemical properties can also be achieved by choosing different monomers for the two zones.

Next, we contrast the mechanical properties of the two zones of the hybrid gel. A typical mechanical test is illustrated in Figure 3 on the dye-soaked gel from Figure 2. The different colors of the two regions help in visualization. In the experiment, the hybrid gel was slowly stretched by hand until it broke. The key result is shown in Figure $3 b$, where we see that the DMAA/BIS portion (green) does not elongate much on stretching; i.e., it is not very elastic or flexible. In comparison, the DMAA/LAP portion (blue) can be stretched to several times its original length. Ultimately, when the hybrid as a whole breaks (Figure 3c), it consistently does so in the middle of the DMAA/BIS region (this was seen in every experimental run). Note that the break does not occur at the interface between the two gel regions, which is usually assumed to be the weakest point in a composite or hybrid. This indicates the high integrity and robustness of the gel/gel interface in our hybrid.

Overall, Figure 3 shows that the DMAA/LAP zone of our hybrid has very different mechanical properties (a much higher strength and extensibility) compared to the DMAA/BIS zone. The higher strength and extensibility of laponite-cross-linked gels are well-known and are attributed to the fact that the polymer chains between junction points in these gels are longer and less polydisperse than those in a conventional gel. ${ }^{17,18}$ Incidentally, we performed the stretching by hand to ensure a gentle grip on the gel. When stretching was attempted with a clamp or vise, the hybrid would prematurely tear at the gripping point of the DMAA/BIS portion, which further shows its lower strength. Also, we should clarify that the same results were obtained on dye-free gels, and so the presence of dye has no influence on the mechanical tests.

Lastly, we focus on the optical heterogeneity of our hybrid gels. It is known that laponite nanodiscs impart optical anisotropy (birefringence) to laponite-cross-linked gels. ${ }^{21,22}$ The birefringence is concentration-dependent. ${ }^{21,22}$ At low laponite concentrations, the nanodisks are oriented randomly and the gel is not birefringent at rest (Figure $4 \mathrm{~b}$ ). However, above a critical laponite concentration $\left(C_{\text {crit }} \approx 6 \mathrm{wt} \%\right)$, the nanodisks stack together into columns, and the resulting alignment of the particles causes the gel to be birefringent at rest. ${ }^{22}$ Birefringence implies that the material shows streaks of light when viewed under crossed polarizer plates. We used this property to engineer hybrid gels with birefringent regions. For these experiments, we worked with NIPA as the monomer because its thermoresponsive property also proves useful in our experiments (see below). We created the hybrid by first forming a pattern or "message" consisting of the letters "UMD" using a pregel mixture of NIPA/LAP with $6.4 \mathrm{wt} \%$ laponite (above $C_{\text {crit }}$ ), as shown in Figure 4a. Thereafter, a pregel mixture of NIPA/LAP with 3.3 wt \% laponite (below $C_{\text {crit }}$ ) was poured around and over the message. The hybrid was then polymerized, and the photograph in Figure $4 \mathrm{~b}$ shows the material after polymerization. Note that the hybrid gel is 
(a) Message hidden in hybrid gel at rest (viewed under crossed-polarizers)

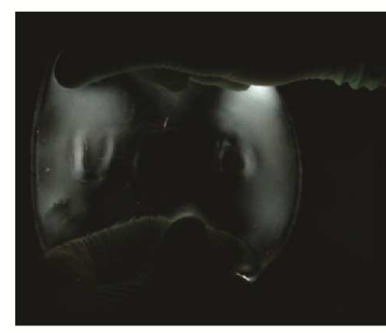

\section{.}

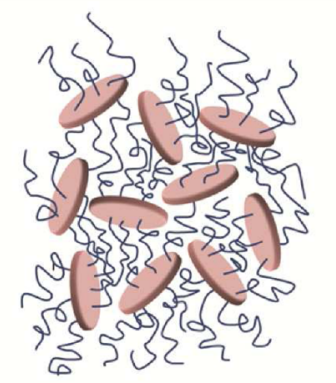

(b) Message seen on stretching due to stress-induced birefringence
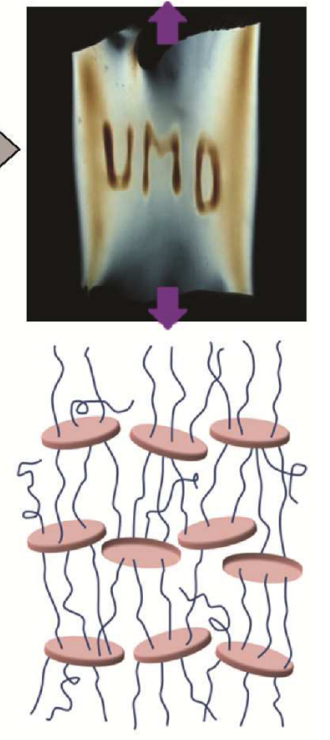

Figure 5. A second example of embedding a hidden pattern or "message" in a hybrid gel. In this case, the message is created with NIPA/LAP (4.9 wt \%), and it is surrounded by NIPA/LAP (3.3 wt \%). When the hybrid is viewed under crossed polarizers, the message is not visible when the gel is at rest, as shown in (a); instead, it is revealed when the gel is uniaxially stretched, as shown in (b). This result exploits the stress-induced birefringence of laponite systems. At rest, the particles are unaligned, as indicated by the schematic in (a). When the gel is stretched, the particles align, as shown by the schematic in (b), which causes birefringence. The message region is more birefringent than the surrounding gel in (b) due to the higher laponite content in the former.

smooth, transparent, and homogeneous, and the message is not discernible. To reveal the hidden message, the hybrid is viewed under crossed polarizer plates, as shown in Figure 4c. The regions corresponding to the message are strongly birefringent compared to the background, allowing the message to be easily read off.

Another way to reveal the hidden message is by heating the hybrid gel above $\sim 32{ }^{\circ} \mathrm{C}$, which is the lower critical solution temperature (LCST) of NIPA. ${ }^{3}$ For example, Figure $4 \mathrm{~d}$ shows the above hybrid immersed in water at $50{ }^{\circ} \mathrm{C}$, and the photograph was taken $30 \mathrm{~s}$ after immersion. In this case, the message can be seen even under direct light. The contrast is apparent between the message region of the gel (6.4 wt \% laponite), which is transparent, and the adjoining gel (3.3 wt \% laponite), which is white and opaque. These differences in opacity arise because the laponite content affects the thermoresponsive character of NIPA gels. When a NIPA gel is heated above its LCST, the polymer segments between crosslink points tend to aggregate and form large clusters, which strongly scatter light and hence lead to opacity. ${ }^{4}$ In the case of NIPA cross-linked with a large concentration of laponite, the segments between adjacent laponite particles are quite short. ${ }^{16,17}$ As a result, there is less aggregation above the LCST and thus less opacity for a gel with $6.4 \mathrm{wt} \%$ laponite. $^{17}$ In comparison, at low laponite content, there is more aggregation of segments when heated above the LCST-thus

a gel with 3.3 wt \% laponite becomes significantly turbid and opaque. ${ }^{17}$

In a variation of the above approach, we created a hybrid gel that reveals its hidden message only when it is subjected to a substantial uniaxial stress (Figure 5). In this case, the message "UMD" was formed by a NIPA/LAP pregel mixture with $4.9 \mathrm{wt}$ $\%$ laponite. This was surrounded as before by a pregel of NIPA/LAP with 3.3 wt \% laponite. Both these laponite concentrations are below $C_{\text {crit }}$ and so there is negligible birefringence anywhere in the gel at rest. Thus, the message is not visible under crossed polarizers (Figure 5a, left panel). However, when subjected to a uniaxial stress, the polymer segments tend to align parallel to the direction of the stress while the laponite disks align perpendicular to it, as shown by the schematics in Figure $5 \mathrm{~b} .{ }^{17,22}$ This causes stress-induced birefringence, which again is higher in gels with higher laponite content. The right panel of Figure 5a shows a photograph of the hybrid gel while it is stretched uniaxially by hand. Both the message region and the surrounding gel show the stressinduced birefringence. Since the message portion has a higher laponite content, it is more birefringent than the surrounding portion, allowing the message to be discerned.

\section{CONCLUSIONS AND OUTLOOK}

We have demonstrated that hybrid hydrogels can be easily created in which individual components retain their identities in a single continuous matrix. Hybrids have been engineered with regions or patterns of one gel juxtaposed with another. Our method to assemble these hybrids is quicker and easier than those used to make traditional interpenetrated networks. Moreover, the gel/gel interfaces in our hybrids have high mechanical integrity and are not the failure points during mechanical testing. To demonstrate the utility of our method, we have developed hybrids of two gels made with the same monomer but different cross-linkers. Regions of the hybrid that were cross-linked by laponite particles had distinct chemical properties (ability to selectively absorb a cationic dye), mechanical properties (higher strength and extensibility), and optical properties (birefringence). We also showed hybrids with an embedded message or pattern that remained hidden until it was revealed by viewing the material under crossed polarizers at rest or under stress. In another example, the hidden pattern was exposed by an external stimulus, i.e., by heating the hybrid above a critical temperature.

Our approach can be extended to create a range of new hybrid gels by varying the monomer and/or the cross-linker for different zones of the gel and also by forming hybrids of numerous gels (i.e., more than just two). Hybrids could have applications in separations, mechanical-optical sensors, biomaterials, drug delivery, and many other areas. We wish to mention a couple of possibilities in this regard. Currently, gels are used as scaffolds for stem cells to differentiate into specific cell types or tissues. ${ }^{8,10}$ It is known that the differentiation is triggered by the physical and chemical nature of the scaffold. Thus, hybrid gels may be useful to induce stem cells to simultaneously differentiate into several different cell types within adjacent regions of a continuous matrix-this would be a step toward the bottom-up assembly of a tissue. ${ }^{10}$ Second, we note that many soft materials in biology and nature are actually hybrids. For example, the spinal disk consists of a soft gel-like core (nucleus pulposus) surrounded by a firmer outer layer (annulus fibrosus), yet the two are integrated into a single, fused material. $^{23,24}$ It is clear that a single polymeric gel cannot 
provide an adequate model for the entire spinal disk. ${ }^{24}$ However, a hybrid gel created by our approach may allow for the first time a faithful replication of the mechanical heterogeneity inherent in a spinal disk.

\section{AUTHOR INFORMATION}

Corresponding Author

*E-mail: sraghava@umd.edu.

Notes

The authors declare no competing financial interest.

\section{REFERENCES}

(1) Tanaka, T. Sci. Am. 1981, 244, 124-138.

(2) Osada, Y.; Gong, J. P.; Tanaka, Y. J. Macromol. Sci., Polym. Rev. 2004, C44, 87-112.

(3) Hirokawa, Y.; Tanaka, T. J. Chem. Phys. 1984, 81, 6379-6380.

(4) Schild, H. G. Prog. Polym. Sci. 1992, 17, 163-249.

(5) Beltran, S.; Baker, J. P.; Hooper, H. H.; Blanch, H. W.; Prausnitz, J. M. Macromolecules 1991, 24, 549-551.

(6) Inomata, H.; Goto, S.; Otake, K.; Saito, S. Langmuir 1992, 8, 687-690.

(7) Klouda, L.; Mikos, A. G. Eur. J. Pharm. Biopharm. 2008, 68, 3445.

(8) Peppas, N. A.; Hilt, J. Z.; Khademhosseini, A.; Langer, R. Adv. Mater. 2006, 18, 1345-1360.

(9) Lee, K. Y.; Mooney, D. J. Chem. Rev. 2001, 101, 1869-1879.

(10) Drury, J. L.; Mooney, D. J. Biomaterials 2003, 24, 4337-4351.

(11) Hu, Z. B.; Zhang, X. M.; Li, Y. Science 1995, 269, 525-527.

(12) Hu, Z. B.; Chen, Y. Y.; Wang, C. J.; Zheng, Y. D.; Li, Y. Nature

1998, 393, 149-152.

(13) Cummins, H. Z. J. Non-Cryst. Solids 2007, 353, 3891-3905.

(14) Haraguchi, K.; Takehisa, T. Adv. Mater. 2002, 14, 1120-1124.

(15) Haraguchi, K.; Takehisa, T.; Fan, S. Macromolecules 2002, 35, 10162-10171.

(16) Haraguchi, K.; Li, H. J.; Matsuda, K.; Takehisa, T.; Elliott, E. Macromolecules 2005, 38, 3482-3490.

(17) Haraguchi, K. Colloid Polym. Sci. 2011, 289, 455-473.

(18) Haraguchi, K.; Li, H. J. Macromolecules 2006, 39, 1898-1905.

(19) Thomas, P. C.; Cipriano, B. H.; Raghavan, S. R. Soft Matter 2011, 7, 8192-8197.

(20) Li, P.; Siddaramaiah; Kim, N. H.; Yoo, G. H.; Lee, J. H. J. Appl. Polym. Sci. 2009, 111, 1786-1798.

(21) Murata, K.; Haraguchi, K. J. Mater. Chem. 2007, 17, 3385-3388.

(22) Haraguchi, K.; Li, H. J.; Song, L. Y.; Murata, K. Macromolecules 2007, 40, 6973-6980.

(23) Boelen, E. J. H.; van Hooy-Corstjens, C. S. J.; Bulstra, S. K.; van Ooij, A.; van Rhijn, L. W.; Koole, L. H. Biomaterials 2005, 26, 66746683.

(24) Nesti, L. J.; Li, W. J.; Shanti, R. M.; Jiang, Y. J.; Jackson, W.; Freedman, B. A.; Kuklo, T. R.; Giuliani, J. R.; Tuan, R. S. Tissue Eng., Part A 2008, 14, 1527-1537. 